\title{
How to manage a spontaneous coronary artery dissection: reconsidering diagnosis and therapy
}

\author{
Dario Buccheri*,1 \\ ${ }^{1}$ Interventional cardiology unit, S. Antonio Abate Hospital, Trapani, Italy \\ *Author for correspondence: Tel./Fax: + 39092380 9177; dariobuccheri@gmail.com
}

First draft submitted: 14 January 2018; Accepted for publication: 6 November 2018; Published online: 22 November 2018

Dear Editor,

I read with great interest the case series recently published by Ahmed et al. [1]. The authors reported a case series in which seven patients were affected by spontaneous coronary artery dissection (SCAD). A particular emphasis was adopted apropos the diagnosis and management of this underestimated entity.

However, here I would like to offer up some food for thought that could be useful and interesting for Future Cardiology readers.

SCAD is a nontraumatic, noniatrogenic detachment of the coronary wall layers that may limit the blood flow causing myocardial ischemia. Young women are primarily affected and clinical manifestations vary from chest pain to sudden cardiac death. It occurs in $0.1-1.1 \%$ of angiographic series and represents $0.1-4 \%$ of acute coronary syndromes (ACS) according to different casuistry reported [2,3]. However, emerging evidence shows that this estimate is under-representative, primarily because its classical angiographic hallmarks are lacking in $>70 \%$ of the angiographies [4] and may be discovered exclusively with intravascular imaging [4,5]. Furthermore, its management remains challenging because of the lack of evidence supporting standard MT, and the role of percutaneous or surgical revascularization is still debated.

Recently, the European Society of Cardiology, in partnership with the Acute Cardiovascular Care Association, established a European SCAD registry as a platform for collaborative research with the aims of improving the knowledge on the topic for an improvement in its management through a shared strategy.

Regarding the initial momentum of the arterial wall separation, two hypotheses have been proposed [3]. First, the 'intimal tear' hypothesis consists of a primitive, noniatrogenic disruption of the intraluminal interface, creating an entry door for blood infiltration in the vessel wall causing an intramural hematoma inside the false lumen, thus leading to arterial wall separation and possibly vessel occlusion. The medial hemorrhage is the second hypothesis in which the primary mechanism leading to dissection is a hemorrhage into the vessel wall that could be due to primitive noniatrogenic rupture or thrombosis of vasa vasorum [3].

A relevant link between SCAD and fibromuscular dysplasia (FMD) was shown by Saw and colleagues who found FMD $\geq 1$ noncoronary territory in $86.0 \%$ of a cohort of SCAD patients. Particularly, $58.1 \%$ had renal FMD, $48.8 \%$ iliac and $46.5 \%$ cerebrovascular whose $14.0 \%$ had intracranial aneurysm [6].

Concerning the diagnosis, in view of the real difficulty confirmed by the high rate of misdiagnosed SCAD [4,5], an interesting and useful score system was previously published $[7,8]$. This system allows interventionists to select the suspected cases in which intravascular imaging, namely optical coherence tomography (OCT), should be recommended as the first choice, thanks to its higher spatial resolution of about ten-times greater than intravascular ultrasound (IVUS), which represents the second line of endovascular imaging that could uncover the presence of a SCAD, thus reducing the time to get the correct diagnosis and a proper therapy.

In this light, our group previously published several cases in which OCT uncovered the presence of SCAD [7,9], above all, type 2 and 3 following Saw angiographic classification [3].

Regarding therapy, in 2017, an experts survey was published [10] in which a medical therapy strategy (MT) was preferred by the majority of those interviewed as first-line treatment in cases of stable patients. During ACS; however, an increasing consensus felt the first choice should be interventional, particularly through the use of

Future Medicine 
drug-eluting stents (DES) followed by bioresorbable vascular scaffold (BVS) implantation. The latter device has the great advantage of the absence of permanent metallic prosthesis that, in this particular setting with the absence of atherosclerotic burst, could represent a highly intriguing strategy allowing the vessel seal. Furthermore, our group suggested an invasive treatment in cases of dissection involving vessel with diameter $\geq 3 \mathrm{~mm}$ or proximal vessel tracts $[7,8]$.

In this view, we previously published our experience on SCAD management successfully with bioresorbable vascular scaffold implantation [7,9] and a rare case of multivessel SCADs in which multiple drug-eluting stents implantation was requested [11].

Apropos MT, the preferred drugs, following experts' opinion should be aspirin, P2Y12 inhibitors, $\beta$-blockers and statins. However, the last drug does not have a universal consensus due to the absence of atheroma and the results of a study that showed a not so useful role in this setting and even more, a potentially harmful role favoring recurrent SCAD [12]. Dual antiplatelet therapy is prescribed usually for 12 months, primarily in the case of stent/scaffold implantation.

Recently, a link between SCAD and Takotsubo Cardiomyopathy (TTC) as nonatherosclerotic causes of myocardial infarction was highlighted. These entities share several common features, although the exact mechanism is yet to be fully established. In fact, they have a predilection for the female gender and, in both, hormonal effects and sympathetic burst associated with a stressful event are known to be involved.

It remains unclear whether SCAD or TTC occurs first, causing the second one. Two main hypotheses have been proposed: first, SCAD could be the stressful event leading to TTC causing the mid-distal and apical akinesia of the left ventricular wall, mimicking the famous Japanese octopus cage during echo or angiography; second, the external torsion forces and mechanical solicitations associated with typical wall motion abnormalities occurring during a TTC could cause the dissection of intima especially in transition segments between the basal hyperkinesis and mid-apical akinesia [13].

Particularly, in the first case of the series by Ahmed et al., for example, the aforementioned scoring system resulted in at least 4 points, according to clinical history and angiographic aspect which suggests the OCT analysis should be performed, as completed by the authors. Once the SCAD diagnosis was discovered, in a case of proximal coronary artery tract or $\geq 3 \mathrm{~mm}$ vessel's diameter, our suggestion is to manage invasively with stent/scaffold implantation [7-9,14].

In this particular case, consisting of ACS setting, the invasive strategy appeared more useful and confident than medical management.

In conclusion, we feel our suggestions could have sped up diagnosis and made the therapy more efficient that, in my opinion, should have been interventional in most cases with stent/scaffold implantation, considering the ongoing ACS. This is made even more important when you consider the potentially life-threatening risk and complications that could occur in very young people, like the patients of the case series reported.

Disclaimer

The opinions expressed in this article are those of the author and do not necessarily reflect the views of Future Medicine Ltd.

Financial \& competing interests disclosure

The author has no relevant affiliations or financial involvement with any organization or entity with a financial interest in or financial conflict with the subject matter or materials discussed in the manuscript. This includes employment, consultancies, honoraria, stock ownership or options, expert testimony, grants or patents received or pending, or royalties.

No writing assistance was utilized in the production of this manuscript.

\section{References}

1. Ahmed MA, Hamra M, Ali M et al. Spontaneous coronary artery dissection, challenges of diagnosis and management. Future Cardiol. 13(6), 539-549 (2017).

2. Yip A, Saw J. Spontaneous coronary artery dissection-a review. Cardiovasc. Diagn. Ther. 5(1), 37-48 (2015).

3. Saw J, Mancini GB, Humphries KH. Contemporary review on spontaneous coronary artery dissection. J. Am. Coll. Cardiol. 68(3), 297-312 (2016).

4. Saw J, Mancini GBJ, Humphries $\mathrm{K}$ et al. Angiographic appearance of spontaneous coronary artery dissection with intramural hematoma proven on intracoronary imaging. Catheter. Cardiovasc. Interv. 87(2), E54-E61 (2016). 
5. Alfonso F, Paulo M, Gonzalo N et al. Diagnosis of spontaneous coronary artery dissection by optical coherence tomography. J. Am. Coll. Cardiol. 59(12), 1073-1079 (2012).

6. Saw J, Ricci D, Starovoytov A et al. Spontaneous coronary artery dissection: prevalence of predisposing conditions including fibromuscular dysplasia in a tertiary center cohort. JACC Cardiovasc. Interv. 6(1), 44-52 (2013).

7. Buccheri D, Piraino D, Latini RA et al. Spontaneous coronary artery dissections: a call for action for an underestimated entity. Int. J. Cardiol. 214, 333-335 (2016).

8. Buccheri D, Piraino D, Andolina G. Score system approach to diagnose and manage spontaneous coronary artery dissection. Rev. Esp. Cardiol. 69(9), 878-879 (2016).

9. Buccheri D, Piraino D, Cortese B. Intravascular imaging as a tool for definite diagnosis of acute coronary syndrome caused by spontaneous coronary artery dissection. Int. J. Cardiol. 214, 43-45 (2016).

10. Buccheri D, Zambelli G, Alfonso F et al. Pulse on spontaneous coronary artery dissections: experience-based survey. JACC Cardiovasc. Interv. 10(14), 1469-1471 (2017).

11. Buccheri D, Milazzo D, Geraci $S$ et al. A lesson from intravascular imaging: insights for recognizing a spontaneous coronary artery dissection. J. Thorac. Dis. 9(12), 5363-5367 (2017).

12. Tweet MS, Hayes SN, Pitta SR et al. Clinical features, management and prognosis of spontaneous coronary artery dissection. Circulation 126(5), 579-588 (2012).

13. Buccheri D, Zambelli G. The link between spontaneous coronary artery dissection and takotsubo cardiomyopathy: analysis of the published cases. J. Thorac. Dis. 9(12), 5489-5492 (2017).

14. Buccheri D, Zambelli G. Focusing on spontaneous coronary artery dissection: actuality and future perspectives. J. Thorac. Dis. 8(12), E1784-E1786 (2016). 
